\title{
Neurocysticercosis Burden in Pig Farming Community of North India
}

\author{
Kashi Nath Prasad \\ Professor, Department of Microbiology, \\ Sanjay Gandhi Postgraduate Institute of Medical Sciences,Lucknow.
}

\begin{abstract}
Neurocysticercosis (NCC) is the most common cause of acquired active epilepsy (AE). $\mathrm{NCC}$ is under reported in India due to lack of systematic systematic studies. We investigated NCC burden in pig farming community of Lucknow district.

Total 294 families with 1640 subjects from 30 villages were surveyed for AE; 595 asymptomatic individuals underwent magnetic resonance imaging of brain. TLR4, MMP9, ICAM1 and GST genes polymorphisms were studied for their role in symptomatic disease. Slaughtered pigs were screened for cysticercosis.
\end{abstract}

Total 95 (5.8\%) subjects with AE were identified; $48.3 \%$ of them had NCC. Ninety $(15 \%)$ asymptomatic individuals had NCC. Thirteen $(26 \%)$ of 50 pigs slaughtered had cysticercosis.

The results showed high NCC burden in pig farming community and NCC as major cause of AE. Individuals with polymorphic TLR4, MMP9, ICAM1 and GST genotypes were susceptible for symptomatic disease. High swine cysticercosis prevalence suggests the transmission dynamic between human and swine in the community.

Keywords: Active epilepsy, host genetic factors, neurocysticercosis, seizure, swine cysticercosis

Correspondence : Prof. KN Prasad, Department of Microbiology, Sanjay Gandhi Postgraduate Institute of Medical Sciences, Lucknow-226014, India. Email: kashinprasad@gmail.com.

DR. PRAN NATH CHHUTTANI ORATION delivered during NAMSCON 2016 at the All India Institute of Medical Sciences, Raipur. 


\section{INTRODUCTION}

Neurocysticercosis (NCC), caused by the larva (cysticercus) of Taenia solium tapeworm is the most common parasitic infection of the central nervous system (CNS). In the developing world, $\mathrm{NCC}$ is identified as the most common cause of acquired active epilepsy (AE); $26.3 \%$ to $53.8 \%$ of $\mathrm{AE}$ in the developing countries are due to NCC (1-3). T.solium infection is a two host neglected zoonotic disease, transmitted between human and pig, and from human to human. Human is the only definite host, harbouring the adult tapeworm in the small intestine. Pig is the natural intermediate host whereas human is the accidental dead end intermediate host. When human consume cysticercotic pork, the cysticercus develops into an adult worm in the intestine. The terminal gravid segment (proglottid) of the adult worm contains thousands of eggs, which are excreted through faeces contaminating the environment. When eggs are ingested by free roaming swine and accidentally by man through contaminated vegetables, food and water, the eggs lose their coat by the action of the gastric acid and pancreatic enzymes, and the hexacanth embryos or oncospheres are liberated. Oncospheres by using their hooklets cross the intestinal wall and through systemic circulation, they reach the different internal organs of the host, like skeletal muscles, CNS, subcutaneous tissue, eye, etc. Now, the oncospheres lose their hooklets, acquire a vesicular shape and evolve into cysticerci by gradual invagination of the protoscolex (invaginated scolex) in several months (4).

NCC is associated with considerable morbidity related to seizures, strokes, hydrocephalus, and long term treatment; however mortality is minimal and frequently associated only with complicated cases of encephalitis, cerebral edema, hydrocephalus with increased intracranial pressure and stroke. The disease is highly endemic in Latin America, Asia, Africa, and especially in countries where pig husbandry is practiced (5). NCC accounts for approximately 2 million cases with 50,000 deaths per year (6). It is now being increasingly reported in developed countries due to increased travel to disease endemic areas and migration of tapeworm carriers or people infected with the disease from endemic areas (7).

The manifestations of NCC are sometimes polymorphic and some individuals with NCC may remain asymptomatic where parasite dies without treatment, while in others it provokes severe neurological disorders like seizures (8). It has been found that the disease is asymptomatic in the initial phase; however, some patients develop fever and headache during tissue invasion by the larvae but most patients do not become symptomatic until 5 to 7 years after initial infection. Asymptomatic period may range from 1 to 30 years or even more $(9,10)$. The true incidence of asymptomatic NCC is unknown because studies defining the ratio between the 
proportion of the population at risk and those actually has the disease are lacking. Nevertheless, various studies from Mexico and South America have found that up to $50 \%$ of patients with evidence of NCC by laboratory testing or neuroimaging are asymptomatic (11). Despite widespread exposure, only few individuals develop symptomatic disease, while others may remain asymptomatic even throughout life but the reasons largely remain unknown. We conducted systematic studies in a rural pig farming community of Mohanlal Ganj block, Lucknow district to estimate NCC burden in symptomatic and asymptomatic individuals, and the possible role of host genetic factors in the development of symptomatic disease. We also examined pigs slaughtered for human consumption for cysticercosis.

\section{Study Subjects:}

Total 294 families from 30 villages were chosen based on 30 cluster sampling approach recommended by World Health Organization. Individuals with AE were identified on door-to-door survey. We also examined stool specimens for eggs of Taenia to determine the taeniasis burden in the community. Patients with symptomatic disease (AE/ seizure) and asymptomatic (with no neurological symptoms) individuals underwent magnetic resonance imaging (MRI) of the brain. Further, we collected blood from NCC related AE patients (symptomatic) and asymptomatic individuals, and studied TLR4, MMP9, ICAM 1 and GST enzyme genes polymorphisms and their expressions to determine their role in the development of symptomatic disease. Pigs slaughtered for human consumption were also examined for cysticercosis by visiting local slaughter houses.

\section{Disease Burden of Taeniasis :}

Demographic, clinical and epidemiological data were collected from 1181 subjects in 210 households in 30 villages. Stool specimens from 924 subjects were examined for eggs of Taenia and other parasites. Eggs and/ or proglottids of Taenia species were detected in $172(18.6 \%)$ individuals. This is one of the highest rates of taeniasis reported in literature. Factors associated with intestinal taeniasis on multivariate analysis were age above 15 years, history of passage of Taenia segments in stool, undercooked pork consumption and poor hand hygiene (12). In an earlier study from Uttar Pradesh, overall prevalence of $\mathrm{T}$. solium taeanisis was $2 \%$ in the community (13). In Chandigarh (India), the prevalence of taeniasis ranged from 0.5 $2 \%$ in hospitalized patients and $12-15 \%$ in labour colonies where pigs were raised (14). In Goa, the estimated prevalence of taeniasis was reported to be $9.7 \%$ (15).

\section{Prevalence of Symptomatic/ Asymptomatic NCC :}

Total 95 of $1640(5.8 \%)$ subjects surveyed in the community had AE; $48.3 \%$ of them had NCC on MRI. Single cyst (cysticercus) was detected in 21 (47.7\%), two cysts in $11(25.0 \%)$ and 

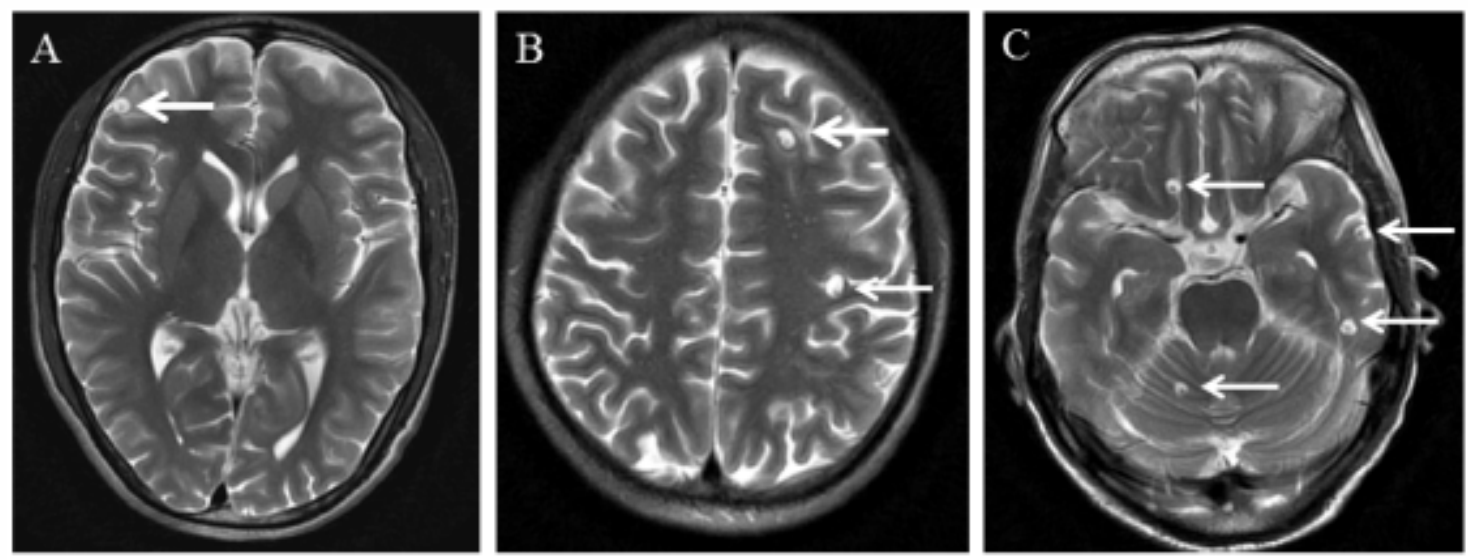

Fig.1 : Magnetic resonance image of symptomatic patients with neurocysticercosis showing cysticerci in the brain parenchyma.T2-weighted axial images at different sections show(A)single cyst, (B)two and (C)multiple cysts.

multiple cysts $(>2)$ in $12(27.3 \%)$ patients. MRI pictures of individuals with single cyst, two and multiple cysts infections are shown in figure 1. Occurrences of vesicular (viable), colloidal/degenerating, calcified and multiple stages of cystic lesions were detected in $2(4.5 \%), 9$ $(20.5 \%), 21(47.7 \%)$ and $12(27.27 \%)$ individuals respectively. The cystic lesions were present in the parenchyma in all the patients; however, two patients with multiple cysts showed a parasite lodged in the subarachnoid space along with parenchyma(16). Epilepsy in the family and no separate place for pig rearing were identified as risks for NCC clustering. The treatment gap for AE in the community was above $90 \%$. The probable reasons for such a high treatment gap were economic, lack of medical facilities, social prejudice and faith in other alternative treatment modalities. The prevalence of NCC related $\mathrm{AE}$ and epilepsy as such in the pig farming community was tremendously high. In the developed world, the prevalence of $\mathrm{AE}$ varies from 3 to 9 per 1000 populations as compared 49 per 1000 populations in Liberia $(17,18)$. In our study the prevalence of $\mathrm{AE}$ was strikingly high (5.8\% i.e. 58 per 1000 populations) and $48.3 \%$ of them had NCC. In South India, the prevalence of NCC related AE varied from $28.4 \%$ to $31 \%(19,20)$.

$$
\text { Total } 90 \text { of } 595 \text { (15\%) }
$$
asymptomatic individuals who underwent MRI had NCC. Single cyst was found in $65(72.2 \%)$ followed by two cysts in 16 $(17.8 \%)$ and multiple cysts in $9(10.0 \%)$ individuals. MRI pictures of asymptomatic individuals with single cyst, two and multiple cysts infections are shown in figure 2. Occurrence of vesicular, degenerating, calcified/healed and multiple stages of cystic lesions were detected in $16(17.8 \%)$, five (5.6\%), 58 $(64.4 \%)$ and $11(12.2 \%)$ individuals 

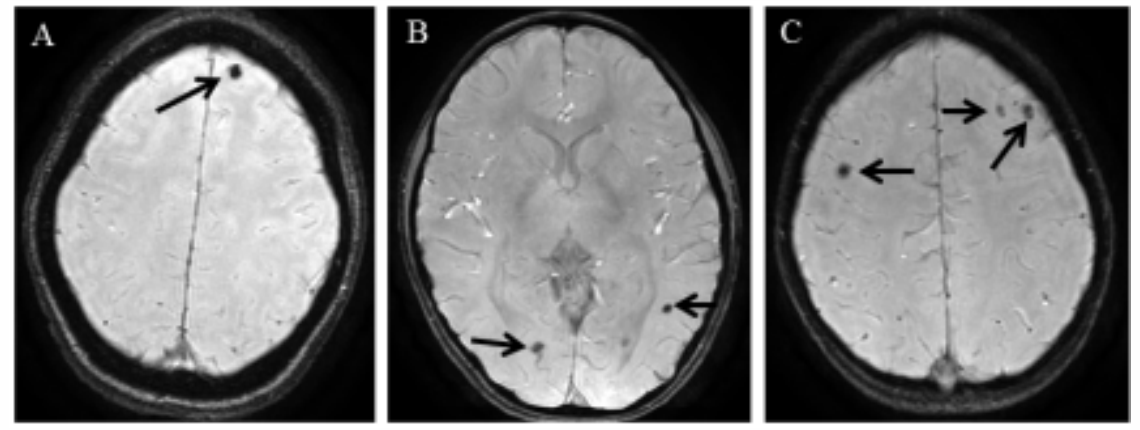

Fig.2 : Magnetic resonance image of asymptomatic neurocysticercosis subjects showing cysticerci in the brain parenchyma. SWAN images at different sections show(A)single cyst, (B)two and (C)multiple cysts.

respectively. The distribution of multiple stages as follows: vesicular and degenerating in two, vesicular and calcified in six, and degenerating and calcified in three cases. Most of the cystic lesions were present in the frontal lobe of the brain $(51.1 \%)$, followed by multiple locations $(14.4 \%)$, occipital $(13.3 \%)$, parietal $(11.1 \%)$ and temporal $(6.7 \%)$ lobes. All the cysts were located in the parenchyma of the brain except one cyst each in ventricle, thalamus and choroids plexus of the brain (21). Comparative distributions of cyst burden according to number, location and stage of the parasite in symptomatic and asymptomatic NCC cases are given in figure 3. A study from Mexico showed that $9.1 \%$ of apparently healthy subjects had calcified NCC lesion, mostly located in the frontal lobe on CT and these individuals were totally symptom free (22).

The number, location and stage wise cyst distributions in symptomatic and asymptomatic subjects were
A

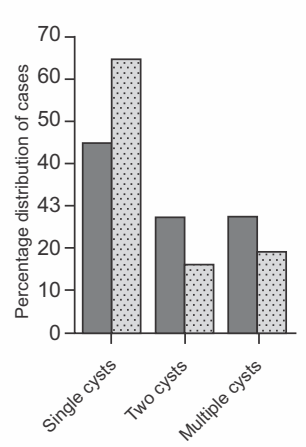

B

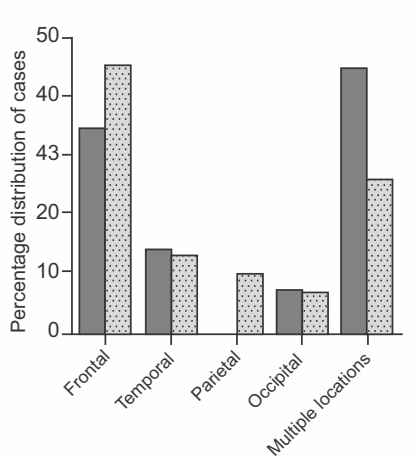

C

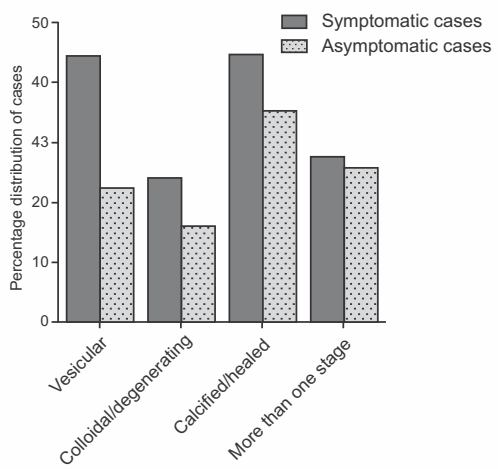

Fig. 3 : Distribution of cysticerci burden in symptomatic vs. asymptomatic NCC cases according to (a) number, (b) location and (c) stage. 
comparable (Figure 3). Now the question arises why some individuals develop symptomatic disease (seizure), while others with similar number, location and stage of the cysticerci remain asymptomatic. Do host genetic factors have some role in the development of symptomatic disease? To answer the above question, we planned to study various host genetic factors and their association with symptomatic disease.

\section{Host Genetic Factors and Symptomatic NCC :}

Most of the human disorders are results of interactions between environmental and genetic factors. Helminths expressed glycans (glycoproteins and glycolipids) may activate host immune cells and some of these molecules can act as specific pathogen associated molecular patterns (PAMPs) for particular TLRs (23). Thomas et al (2003) showed that helminthglycans could act as PAMPs for inducing potent Th2 response via TLR4 dependent mechanism(24). Since studies are lacking, we investigated the role of TLR4Asp299Gly and Thr399Ile polymorphisms and their role in the development of symptomatic NCC.

Our results showed that TLR4 Asp299Gly and Thr399Ile were significantly associated with the occurrence of NCC and progression to symptomatic disease compared to healthy control and asymptomatic NCC subjects. Haplotype Gly/Thr was observed as a risk factor for susceptibility to NCC. Gly and
Ile carriers had significant association with symptomatic NCC (25).

Our earlier studies showed significantly enhanced expressions of MMP-9 and ICAM-1 in symptomatic NCC patients $(26,27)$. We further evaluated the MMP-9 and ICAM-1 gene polymorphisms in NCC subjects. The analysis of the genotype frequency of MMP-9 (R279Q) polymorphism revealed a significant association of the heterozygous genotype (R/Q) with symptomatic subjects compared to asymptomatic subjects. However, there was no significant difference in the genotype frequency between asymptomatic NCC subjects and controls (28). In ICAM-1 K469E polymorphism study, homozygous (EE) variant genotype was associated with 11 and 4 folds increased risk of symptomatic disease when compared with healthy controls and asymptomatic NCC subjects respectively. However no such association was found between healthy controls and asymptomatic NCC subjects. Further, the $\mathrm{E}$ allele had significant association with symptomatic NCC than controls and asymptomatic NCC subjects. The K allele was more prevalent among the healthy controls and showed protective association when compared with symptomatic NCC subjects (29).

Previously we reported higher expression of pro-inflammatory cytokines in symptomatic NCC patients (30). Inflammatory cells with production of inflammatory cytokines activate cellular oxidant-generating pathways. Reactive 
oxygen species (ROS) that are generated during inflammatory conditions are highly reactive and can cause damage to cellular macromolecules such as lipids, DNA and proteins (31). To counteract the damage from ROS, the cells are endowed with a glutathione antioxidant system, which includes glutathione reductase, glutathione peroxidase, and glutathione Stransferases (GSTs). These enzymes maintain redox balance and protect cells from oxidative damage (32). In humans, the GST enzymes can be divided into five main classes: alpha (GSTA), mu (GSTM), pi (GSTP), theta (GSTT), and zeta (GSTZ) that are critical in protection of cells from ROS during inflammatory condition (33-35). These GSTs are polymorphic in nature with reported interindividual variations in their enzymatic activity and level. However, the role of GSTs in NCC remains unexplored. We investigated the polymorphisms in GST genes and their expressions and activities in symptomatic and asymptomatic NCC subjects.
Human GST gene polymorphisms study revealed that the individuals carrying the deletions of GSTM1 and GSTT1 (null genotypes) were at risk for developing symptomatic NCC disease. Genetic variants of GSTM3 and GSTP1 were not associated with symptomatic disease. The total GST activity and levels of GSTM1, GSTT1, and GSTM3 were significantly higher in asymptomatic subjects than in symptomatic and healthy controls. Lower GST activity was observed in individuals with GSTM1 and GSTT1 deletions. The study suggests that the individuals with GSTM1 and GSTT1 deletions are at higher risk to develop symptomatic disease because such individuals have low level of GSTs that fail to protect tissues from ROS related oxidative damage. The higher GST activity and levels of GSTM1, GSTT1, and GSTM3 are likely to protect tissue from oxidative damage thus maintaining asymptomatic condition (36).
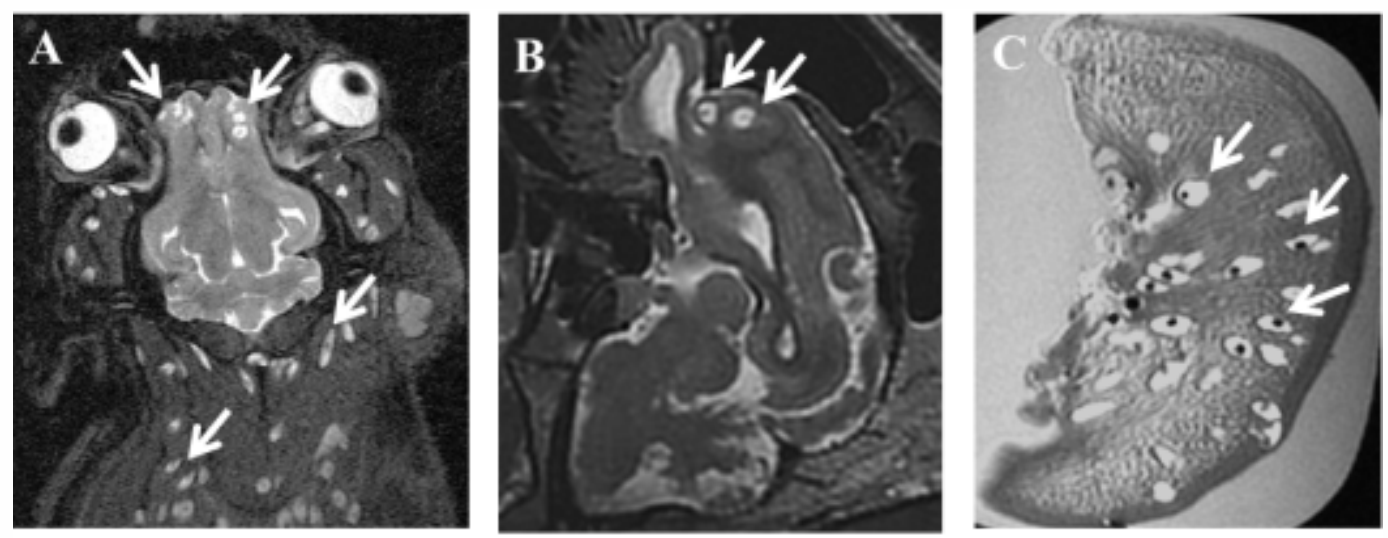

Fig. 4 :T2-weighted magnetic resonance images of pig brain( $A$ and $B$ ), and tongue (C) showing large number of cysticerci (arrowheads). 


\section{Prevalence of Swine NCC :}

Our slaughter housesurvey showed that $13(26 \%)$ of 50 pigs slaughtered for human consumption had cysticerci in their muscles; $5(38 \%)$ and 3 $(23 \%)$ of them also had cysticerci in the brain and liver respectively (37).Further, we developed MRI protocol to detect neurocysticercosis in swine for the first time (38). We also identified several clinical signs of swine NCC and these signs (excessive salivation, excessive blinking and tearing, and subconjunctival nodule) were reported for the first time in literature(39). Other Indian studies showed that $8-10 \%$ of pigs slaughtered for human consumption in Chandigarh and $7 \%$ in Kolkata had muscle cysticercosis $(14,40)$. MRI of pig brain and tounge with cysticerci are shown in figure 4 .

\section{Conclusion :}

The study shows high prevalence of taeniasis and NCC related AE in the pig farming community of North India with tremendous treatment gap. Individuals with polymorphic genotypes of TLR4, MMP9, ICAM1 and GST are more susceptible to develop symptomatic disease (seizure episodes). High human taeniasis and swine cysticercosis prevalence suggests the dynamic of transmission cycle in the community between human and pig. Since cysticercosis is a preventable disease, appropriate measures like health education, improved sanitation, mass awareness, better medical facilities, mass treatment of Taenia carriers, and restriction on sale of measly pork may help to reduce the disease burden and economic loss to the country.

\section{REFERENCES :}

1. Del Brutto OH, Santibanez R, Idrovo L, et al. (2005). Epilepsy and neurocysticercosis in Atahualpa: a door-to-door survey in rural coastal Ecuador. Epilepsia 46: 583-587.

2. Montano SM, Villaran MV, Ylquimiche L, et al. (2005). Neurocysticercosis: association between seizures, serology, and brain CT in rural Peru. Neurology 65: 229-233.

3. Nicoletti A, Bartoloni A, Sofia $\mathrm{V}$, et al. (2005). Epilepsy and neurocysticercosis in rural Bolivia: a population-based survey. Epilepsia 46: 1127-1132.

4. Escobar A, Neito D (1972). Parasitic diseases. In: Pathology of the Nervous System, Minckler J (ed), McGraw-Hill, New York, 25032521.

5. Mayta H, Gilman RH, Prendergast E, Castillo JP, Tinoco YO, Garcia $\mathrm{HH}$, Gonzalez AE, Sterling CR, and for the Cysticercosis Working Group in Peru (2008). Nested PCR for specific diagnosis of Taenia solium taeniasis. J Clin Microbiol 46: 286-289. 
6. Roman G, Sotelo J, Del Brutto O, et al. (2000). A proposal to declare neurocysticercosis an international reportable disease. Bull WHO 78:399-406.

7. Burneo JG, Plener I, Garcia HH, Neurocysticercosis and Epilepsy Research Network( 20009$)$. Neurocysticercosis in a patient in Canada. CMAJ 180: 639-642.

8. Fleury A, Gomez T, Alvarez I, et al. (2003). High prevalence of calcified silent neurocysticercosis in a rural vi 11 a g e of $\mathrm{M}$ e $\mathrm{x}$ i c o . Neuroepidemiology 22:139-145.

9. Dixon HBF, Lipscomb FM (1961). Cysticercosis: an analysis and follow-up of 450 cases. London: Privy Council, Medical Research Council, 1-58.

10. Kurrein F, Vickers AA (1977). Cysticercosis of spine. Ann Trop Med Parasitol 71: 213-217.

11. Chavarria A, Roger B, Fragoso G, et al. (2003). TH2 profile in asymptomatic Taenia solium human neurocysticercosis. Microbes Infect 5: 1109-1115.

12. Prasad KN, Prasad A, Gupta RK, Pandey CM, Singh U (2007). Prevalence and associated risk factors of Taenia solium taeniasis in a rural pig farming community of North India. Trans R Soc Trop Med Hyg 101: 1241-1247.
13. Pathak KM, Gaur SN (1989). Prevalence and economic implications of Taenia solium taeniasis and cysticercosis in Uttar Pradesh State of India. Acta Leiden 57:197-200.

14. Mahajan RC, Chopra JS, Ganguly NK (1982). Human cysticercosis and epilepsy: a serological study. In :Cysticercosis: Present State of Knowledge and Perspectives. Flisser A, Willms K, Laclette JP, Larralde C, Ridaura C, Beltran F(eds), New York: Academic Press, 171-178.

15. Vora SH, Motghare DD, Ferreira AM, Kulkarni MS, Vaz FS (2008). Prevalence of human cysticercosis and taeniasis in rural Goa, India. $J$ Commun Dis 40:147-150.

16. Prasad KN, Prasad A, Gupta RK, et al. (2009). Neurocysticercosis in patients with active epilepsy from pig farming community of Lucknow district, North India. Trans $R$ Soc Trop Med Hyg 103: 144-150.

17. Rocca WA, Savettieri G, Anderson DW, et al. (2001). Sicilian NeuroEpidemiologic Study (SNES) Group. Door-to-door prevalence survey of epilepsy in three Sicilian municipalities. Neuroepidemiology 20:237-241. 
18. Gerrits C (1994). Epilepsy care in a non-clinical setting-a medicalanthropological study among the Bassa and Kpelle in the rain-forest of Liberia, West Africa. Trop Geogr Med 46(Suppl 3):S13-S17.

19. Rajshekhar VM, Raghava V, Prabhakaran V, Ommen A, Muliyil J (2006). Active epilepsy as an index of burden of neurocysticercosis in Vellore district, India. Neurology 67: 2135-2139.

20. Sawhney IM, Lekhra OP, Shashi JS, Prabhakar S, Chopra JS (1996). Evaluation of epilepsy management in a developing country: a prospective study of 407 patients. Acta Neurol Scand 94: 19-23.

21. Prasad KN, Verma A, Srivastava S, Gupta RK, Pandey CM, Paliwal VK (2011). An epidemiological study of asymptomatic neurocysticercosis in a pig farming community in northern India. Trans $R$ Soc Trop Med Hyg 105:531-536.

22. Fleury A, Hernández M, Fragoso G, Parkhouse RME, Harrison LJS, Sciutto E (2003). Detection of secreted cysticercal antigen: a useful tool in the diagnosis of inflammatory neurocysticercosis. Trans $R$ Soc Trop Med Hyg 97: 542-546.
23. Mishra BB, Mishra PK, Teale JM (2006). Expression and distribution of Toll like receptors in the brain during neurocysticercosis. $J$ Neuroimmunol 181:46-56.

24. Thomas PG, Carter MR, Atochina $\mathrm{O}$, et al. (2003). Maturation of dendritic cell 2 phenotype by a helminth glycan uses a toll like receptor 4 dependent mechanisms. $J$ Immunol 171:5837-5841.

25. Verma A, Prasad KN, Gupta RK, et al. (2010). Toll-like receptor 4 polymorphism and its association w i t h s y m t o m a t i c neurocysticercosis. $J$ Infect Dis 202:1219-1225.

26. Verma A, Prasad KN, Nyati KK, et al. (2011). Association of MMP-2 and MMP-9 with clinical outcome of neurocysticercosis. Parasitology 138:1423-1428.

27. Prasad A, Prasad KN, Gupta RK, Pradhan S (2009). Increased expression of ICAM-1 among symptomatic neurocysticercosis. $J$ Neuroimmunol 206:118-120.

28. Gupta RK, Awasthi R, Rathore RK, et al. (2012). Understanding epileptogenesis in calcified neurocysticercosis with perfusion MRI. Neurology 78:618-625. 
29. Singh A, Singh AK, Singh SK, Paliwal VK, Gupta RK, Prasad KN (2014). Association of ICAM-1 K469E polymorphism with ne urocysticercosis. $J$ Neuroimmunol 276:166-171.

30. Verma A, Prasad KN, Cheekatla SS, Nyati KK, Paliwal VK, Gupta RK (2011). Immune response in symptomatic and asymptomatic neurocysticercosis. Med Microbiol Immunol 200:255-261.

31. Arteel GE (2003). Oxidants and antioxidants in alcohol-induced liver disease. Gastroenterology 124:778-790.

32. Limon-Pacheco JH, Gonsebatt ME (2010). The glutathione system and its regulation by neurohormone melatonin in the central nervous system. Cent Nerv Syst Agents Med Chem 10:287-297.

33. Beckett GJ, Hayes JD (1993). Glutathione S-transferases: biomedical applications. Adv Clin Chem 30:281-380.

34. Birben E, Sahiner UM, Sackesen C, Erzurum S, Kalayci O (2012). Oxidative stress and antioxidant defense. World Allergy Organ $J$ 5:9-19.
35. $\mathrm{B}$ o a r d $\mathrm{P} \mathrm{G}, \mathrm{B}$ a k e r R T, Chelvanayagam $\mathrm{G}$, Jermiin LS (1997). Zeta, a novel class of glutathione transferases in a range of species from plants to humans. Biochem J 328:929-935.

36. Singh A, Prasad KN, Singh AK, et al. (2016). Human glutathione Stransferase enzyme gene polymorphisms and their association with neurocysticercosis. Mol Neurobiol DOI 10.1007/ s12035-016-9779-4 (in press).

37. Prasad KN, Chawla S, Jain D, et al. (2002). Human and porcine Taenia solium infection in rural north India. Trans R Soc Trop Med Hyg 96: 515516.

38. Chawla S, Husain N, Kumar S, Pal L, Tripathi M, Gupta RK(2004). Correlative MRI imaging and histopathology in porcine neurocysticercosis. J Magn Reson Imaging 20: 208-215.

39. Prasad KN, Chawla S, Prasad A, Tripathi M, Husain N, Gupta RK (2006). Clinical signs for identification of neurocysticercosis in swine naturally infected with Taenia solium. Parasitol Int 55:151154.

40. Ratnam S, Khanna PN, Bandopadhyaya AK (1983). Incidence of taeniasis in man. Indian J Public Health 27: 70-74. 